Supporting information for

\title{
Design Strategies for High-Efficiency Ratiometric \\ Two-Photon Excited Fluorescent and Room-Temperature Phosphorescent Probes for Hypochlorous Acid
}

Xue-Li Hao,${ }^{\dagger}$ Jing-Fu Guo,${ }^{\ddagger}$ Teng-Fei He,${ }^{\dagger}$ Pan-Pan Lin ${ }^{\dagger}$, Lu-Yi Zou ${ }^{\dagger}$, Li-Bo Yu ${ }^{\dagger}$, Xue-Hui Guo, ${ }^{\dagger}$

Ai-Min Ren, ${ }^{* \dagger}$

${ }^{\dagger}$ Laboratory of Theoretical and Computational Chemistry, Institute of Theoretical Chemistry, Jilin University,

Liutiao Road \#2, Changchun 130061

†School of Physics, Northeast Normal University, 130024, P.R.China

*Corresponding author: Ai-Min Ren

Tel.: +86 $43188498961 ; \quad$ Fax: +86 43188945942 ;

E-mail addresses: aimin_ren@yahoo.com 


\section{Table of Contents}

\section{Theoretical Methods}

1. Two-Photon Absorption

2. The Fluorescence Quantum Yield

\section{Electronic structures, absorption and fluorescent emission properties}

3. Figure S1. Structures and corresponding names of the investigated molecules in water solvent

4. Figure S2. The frontier molecular orbital levels for studied compounds in ground state

5. Figure S3. Electron density difference analysis during electron excitation

6. Figure S4. Contour surfaces of the frontier molecular orbitals for investigated compounds in the excited states

7. Figure S5. Huang-Rhys (HR) and crucial displacement vectors for the normal modes with large HR in low-frequency regime $\left(<1000 \mathrm{~cm}^{-1}\right)$ for all coumarin-based compounds

8. Figure S6. Structures and corresponding names of the experimental molecules

9. Table S1. Calculated one-photon absorption properties of experimental molecules by TD-DFT methods using different functionals

10. Table S2. Calculated two-photon absorption properties of experimental molecules by TD-DFT methods using different functionals

11. Table S3. Calculated fluorescent emission properties of experimental molecules by TD-DFT methods using different functionals

12. Table S4. Fluorescence properties of all the studied compounds

13. Table S5. The singlet-triplet excited states properties of all the studied compounds and spin-orbit coupling matrix elements between $S_{1}$ and $T_{2}$ states

14. Table S6. The first triplet excited state $T_{1}$ properties of all the studied compounds and spin-orbit coupling matrix elements between $T_{1}$ and $S_{0}$ states

15. Table S7. TPA tensor elements $\left(S_{a b}\right)$ and TPA cross section $\left(\sigma^{\mathrm{T}}{ }_{\max }\right)$ of all investigated compounds are calculated using DALTON program in water

16. Table S8. TPA tensor elements $\left(S_{a b}\right)$ and TPA cross section $\left(\sigma^{\mathrm{T}}{ }_{\max }\right)$ of all investigated compounds are calculated using few-state models in water 


\section{THEORETICAL METHODS}

\section{Two-Photon Absorption}

Two-photon absorption intensity is determined by two-photon absorption cross $\left(\sigma^{T P A}\right)$, which is connection with two-photon transition probability $\left(\delta_{a . u .}\right)$ and can be calculated by:1,2

$$
\sigma^{T P A}=\frac{4 \pi^{2} \alpha a_{0}^{5} \omega^{2}}{15 c \Gamma} \delta_{a . u}
$$

Here, $\alpha, a_{0}$ and $c$ respectively are the fine structure constant, Bohr radius and speed of light; $\omega$ represents the photon energy in atomic units and $\Gamma$ is the broadening factor, which describes the spectral broadening of an excitation. ${ }^{3} \delta_{\text {a.u. }}$ can be expressed as: ${ }^{4,5}$

$$
\delta_{a . u}=\frac{1}{30} \sum_{a b}\left(F S_{a a} \bar{S}_{b b}+G S_{a b} \bar{S}_{a b}+H S_{a b} \bar{S}_{b a}\right)
$$

Where, $a, b \in\{x, y, z\}$; The value of $F, H$ and $G$ are 2,2 and 2 for linearly polarized light, and those are $-2,3$ and 2 for the circular case, respectively. Under the electric dipole approximation, the TPA transition tensor $S^{i f}$ between the initial state $i$ and the final state $f$ can be defined as: ${ }^{2}$

$$
S_{a b}^{i f}\left(\omega_{1}, \omega_{2}\right)=\sum_{n \neq i}\left\{\frac{\left\langle i\left|\mu_{a}\right| n\right\rangle\left\langle n\left|\bar{\mu}_{b}\right| f\right\rangle}{\omega_{n i}-\omega_{1}}+\frac{\left\langle i\left|\mu_{b}\right| n\right\rangle\left\langle n\left|\bar{\mu}_{a}\right| f\right\rangle}{\omega_{n i}-\omega_{2}}\right\}
$$

Where, $\left\langle i\left|\mu_{a}\right| n\right\rangle$ represents the $a$-th component of the transition dipole moment, which is between the initial electric state $i$ and intermediate state $n$, and $\omega_{i n}$ is the associated excitation energy. In addition, $\omega_{1}$ and $\omega_{2}$ are the energies of the two photons. If the excitation process is contributed mainly by two different states viz., the ground state and the final state, the equation above can be reduced to:

$$
S_{a b}^{0 f}\left(\omega_{1}, \omega_{2}\right)=\frac{\mu_{a}^{0 f} \Delta \mu_{b}}{\omega_{0 f}-\omega_{1}}+\frac{\mu_{b}^{0 f} \Delta \mu_{a}}{\omega_{0 f}-\omega_{2}}
$$


There, the $\mu_{a} 0 f$ and $\Delta \mu_{a}$ respectively represent the $a$-th component of transition dipole moment from the ground to the $f$ excited state and difference between the excited and the ground state dipole moments. These physical parameters including the TPA transition tensor $\left(S^{i f}\right)$, TPA transition probability $\left(\delta_{\text {a.u. }}\right)$ and TPA cross section $\left(\sigma^{\mathrm{TPA}}\right)$ also can be calculated with the help of quadratic response theory by DALTON program $^{6}$. 


\section{The Fluorescence Quantum Yield}

The fluorescence quantum yield is determined by radiative decay rate $\left(\mathrm{K}_{\mathrm{r}}\right)$ and non-radiative decay rate $\left(\mathrm{K}_{\mathrm{nr}}\right)$, which can be expressed as:

$$
\Phi=\frac{K_{r}}{K_{r}+K_{n r}}
$$

The spontaneous fluorescent emission rate $\mathrm{K}_{\mathrm{r}}$ can be obtained using Einstein transition

probabilities: $^{7} \quad K_{r}=\frac{2 f E^{2}}{c^{3}}$

Here, $f$ is the oscillator strength, $E$ is the transition energy and $c$ is the speed of light.

If the vibronic coupling and harmonic oscillator approximation are taken into account, the $\mathrm{K}_{\mathrm{r}}$ can be calculated according to the formula: ${ }^{8}$

$$
k_{r(i 0 \rightarrow f a)}=\frac{64 \pi^{4}}{h^{4} c^{3}}|\mu|^{2} \sum_{a}\left(v_{i f}+\sum_{j} a_{j} v_{j}\right)^{3} \prod_{j} \frac{S_{j}^{a_{i}}}{a_{j} !} e^{-S_{j}}
$$

Where, $h$ is the Plank constant, $c$ is the speed of light, $\mu$ is the electric transition dipole moment and $S_{j}$ is the Huang-Rhys factor for the $j$-th mode. The frequency of transition from the initial state to final state $v_{i 0 \rightarrow f a}$ can be expressed as: $v_{i 0 \rightarrow f a}=v_{i f}+\sum_{j} a_{j} v_{j}$.

Based on first-order perturbation theory, the phosphorescent radiative decay rate $\mathrm{K}_{\mathrm{r}}^{\mathrm{T}}$ can be expressed as: ${ }^{9}$

$$
K_{\mathrm{r}}^{T_{1}^{\alpha}}{ }_{\left(T_{1}^{\alpha} \rightarrow S_{0}\right)}=\frac{8 \pi^{2} \eta E\left(T_{1}\right)^{3}}{3 \varepsilon_{0} \mathrm{~h}} \sum_{\mathrm{j} \in x, y, z}\left|\sum_{m} \frac{\left\langle T_{1}^{\alpha}\left|H_{s o c}\right| S_{m}\right\rangle}{E\left(S_{m}\right)-E\left(T_{1}\right)} M_{S_{m, j}}\right|^{2}
$$

Here, $\boldsymbol{\eta}$ is refractive index of solvent; $E\left(\mathrm{~T}_{1}\right)$ and $E\left(\mathrm{~S}_{\mathrm{m}}\right)$ are the energies of $\mathrm{T}_{1}$ and $\mathrm{S}_{\mathrm{m}}$ excited states; $\left\langle T_{1}^{\alpha}\left|H_{\text {soc }}\right| S_{m}\right\rangle$ is the spin-orbit matrix elements between three sublevels $(\boldsymbol{\alpha}=\mathrm{x}, \mathrm{y}, \mathrm{z})$ of $\mathrm{T}_{1}$ and $\mathrm{S}_{\mathrm{m}}$ states; $M_{S_{m, j}}$ is the $\mathrm{j}$-axis projection of transition dipole moment from $S_{m}$ to $S_{0}$. At room temperature, the three sublevels of $T_{1}$ are 
determined by thermal population distribution according to Boltzmann statistics. The total decay rate of $\mathrm{T}_{1}$ can be expressed as: $K_{\mathrm{r}\left(T_{1} \rightarrow S_{0}\right)}^{T_{1}}=\frac{1}{3} \sum_{\alpha} K_{\mathrm{r}}^{T_{\alpha}^{1}}$

As for non-radiative decay, it includes internal conversion rate $\left(\mathrm{K}_{\mathrm{ic}}\right)$ from $\mathrm{S}_{1}$ to $\mathrm{S}_{0}$, intersystem crossing rate $\left(\mathrm{K}_{\mathrm{isc}}\right)$ and reverse intersystem crossing rate $\left(\mathrm{K}_{\text {risc }}\right)$ between the singlet and triplet excited states. The internal conversion $\left(\mathrm{K}_{\mathrm{ic}}\right)$ is defined as: ${ }^{10}$

$k_{i c}=\frac{2 \pi}{\mathrm{h}}\left|H_{f i}^{\prime}\right|^{2} \delta\left(E_{f i}+E_{f v_{f}}-E_{i v_{i}}\right)$

Here, the non-Born-Oppenheimer coupling can be described as: ${ }^{11}$

$H_{f i}^{\prime}=-\mathrm{h}^{2} \sum_{l}\left\langle\Phi_{f} \Theta_{f v_{f}} \mid \frac{\partial \Phi_{i}}{\partial Q_{f l}} \frac{\partial \Theta_{i v_{i}}}{\partial Q_{f l}}\right\rangle$

Under the Condon approximation, the eq (9) is expressed as: ${ }^{12}$

$H_{f i}^{\prime}=\sum_{l}\left\langle\Phi_{f}\left|\oiint_{f l}\right| \Phi_{i}\right\rangle\left\langle\Theta_{f v_{f}}\left|\oiint_{f l}\right| \Theta_{i v_{i}}\right\rangle$

Then, the eq (10) is inserted into eq (8) and the delta function is Fourier transformed, we can get that: ${ }^{13}$

$$
k_{i c, k l}=\frac{1}{\mathrm{~h}^{2}} R_{k l} \int_{-\infty}^{+\infty} e^{i \omega_{i f} t} Z_{i v}^{-1} \rho_{i c, k l}(t, T) d t \quad \text { (12) } \quad R_{k l}=\left\langle\Phi_{f}\left|\boldsymbol{P}_{f k}\right| \Phi_{i}\right\rangle\left\langle\Phi_{i}\left|\boldsymbol{P}_{f l}\right| \Phi_{f}\right\rangle
$$

Where, $Z_{i v}{ }^{-1}$ and $\rho_{i c, k l}(\mathrm{t}, \mathrm{T})$ respectively are the partition function and thermal vibration correlation and these physical parameters can be obtained using MOMAP program ${ }^{14}$.

Besides, the intersystem crossing $\left(\mathrm{K}_{\mathrm{isc}}\right)$ and reverse intersystem crossing decay rates $\left(\mathrm{K}_{\mathrm{risc}}\right)$ can be evaluate using semiclassical Marcus equation expressed as: ${ }^{15}, 16$

$$
K_{\mathrm{r}(\text { isc })}=\frac{2 \pi}{\mathrm{h}}\left|\mathrm{H}_{\mathrm{soc}}\right|^{2} \frac{1}{\sqrt{4 \pi \lambda k_{B} T}} \exp \left[-\frac{\left(\lambda \pm\left|\Delta E_{S T}\right|\right)^{2}}{4 \lambda k_{B} T}\right]
$$

Where, $+(-)$ sign is connection with $\mathrm{K}_{\text {risc }}\left(\mathrm{K}_{\text {isc }}\right)$. 
2030

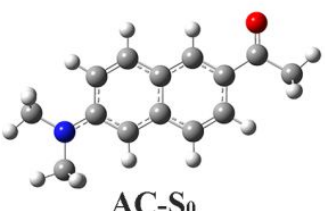

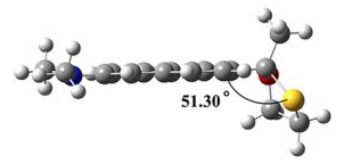

AOCL-So-side view

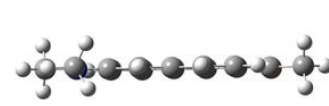

AC-So-side view

$\int^{30}$

COCL-S

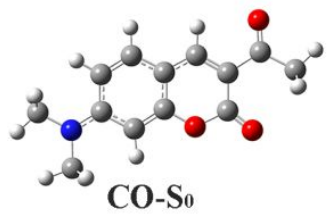

,

${ }^{3}$

benza1-COCL-S

طenza1-CO-S

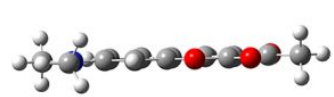

CO-So-side view

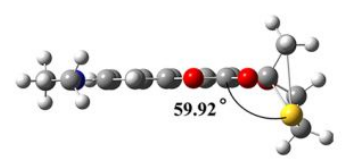

COCL-So-side view
${ }^{30.050}$

AOCL-S1

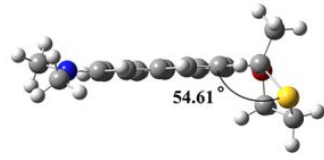

AOCL-S1-side view

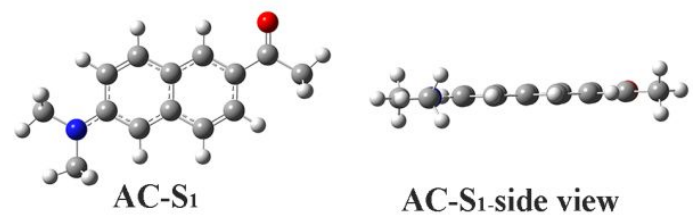

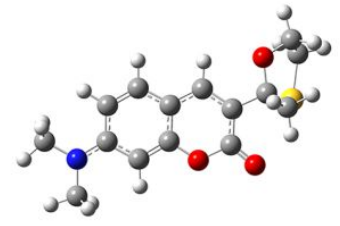

COCL-S1

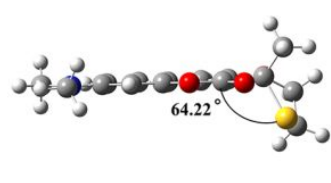

COCL-S1-side view

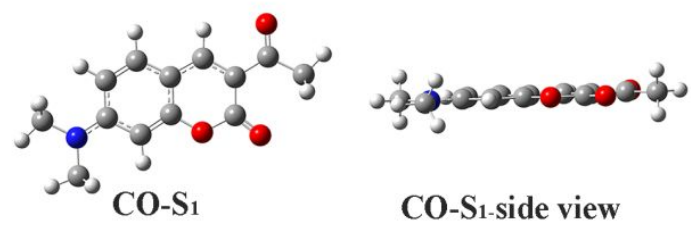

CO-S1-side view

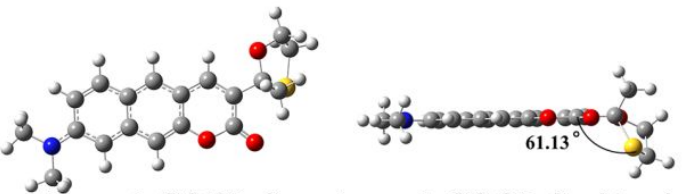

benza1-COCL-S1-side view

benza1-COCL-S 1

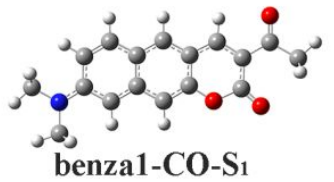

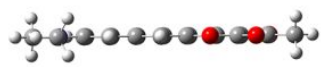
benza1-CO-S1-side view

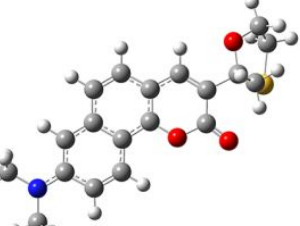

, s,

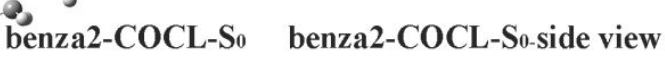

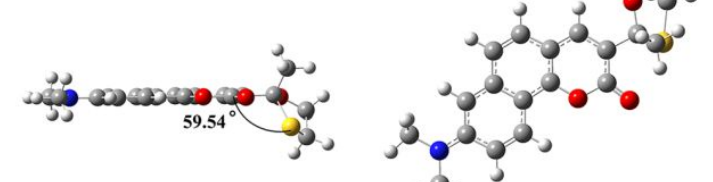

ر
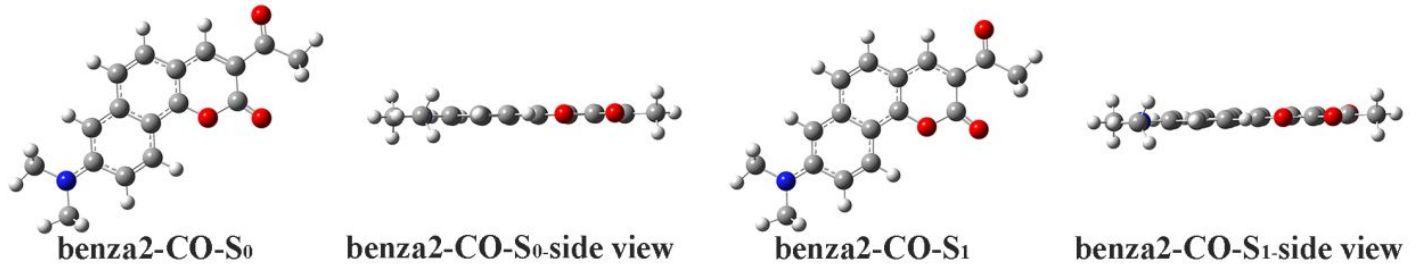

Figure S1. Structures and corresponding names of the investigated molecules in water solvent 


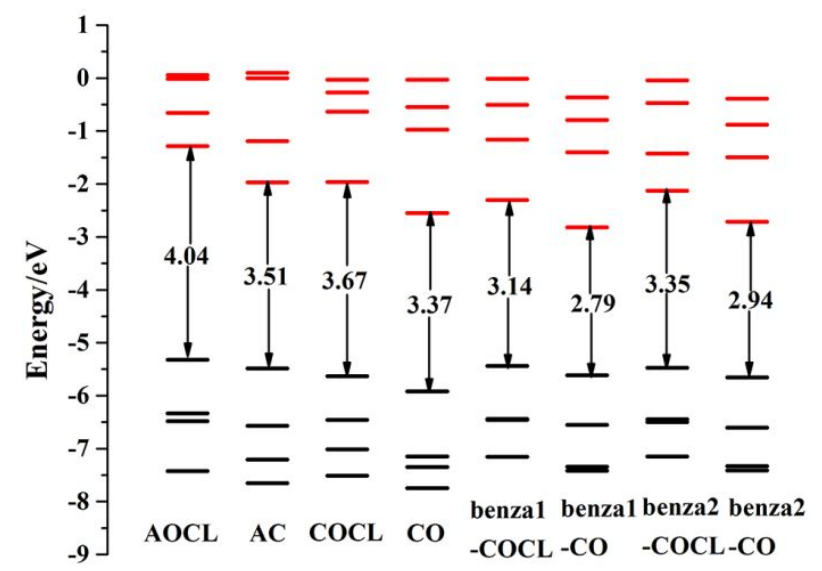

Figure S2. The frontier molecular orbital levels for studied compounds in ground state

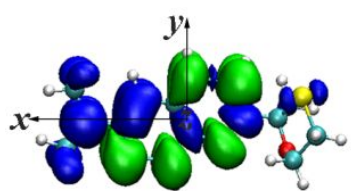

AOCL-S1 front view

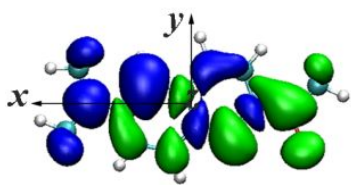

AC-S1 front view

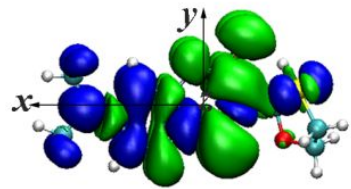

COCL-S1 front view
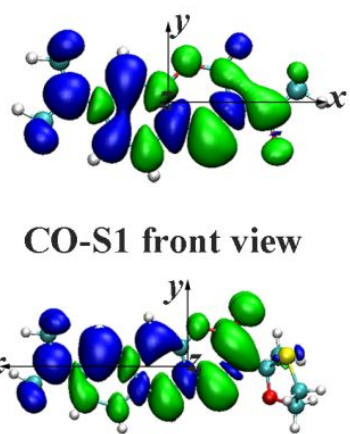

benza1-COCL-S1 front view

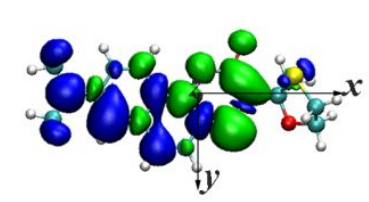

benza2-COCL-S1 front view

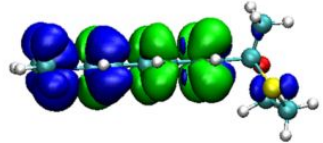

AOCL-S1 side view

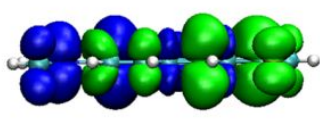

AC-S1 side view

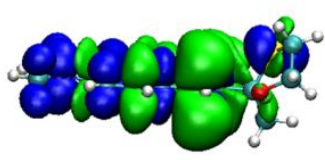

COCL-S1 side view

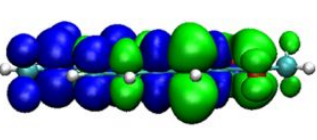

CO-S1 side view

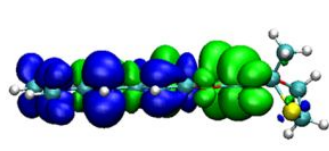

benza1-COCL-S1 side view

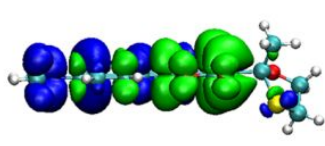

benza2-COCL-S1 side view

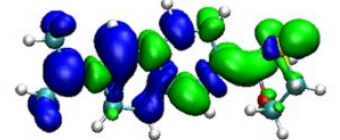

AOCL-S2 front view AOCL-S2 side view
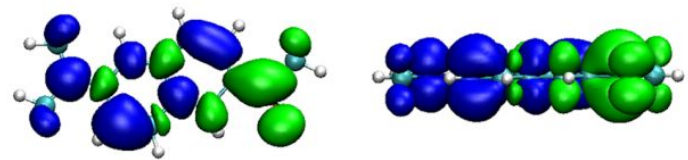

AC-S4 front view

AC-S4 side view
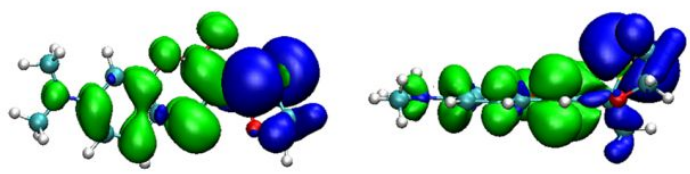

COCL-S2 front view

COCL-S2 side view
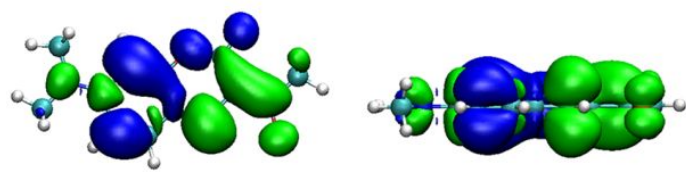

CO-S3 front view

CO-S3 side view
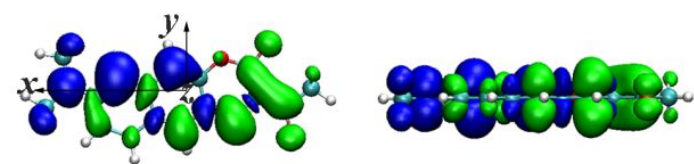

benza1-CO-S1 front view

benza1-CO-S1 side view

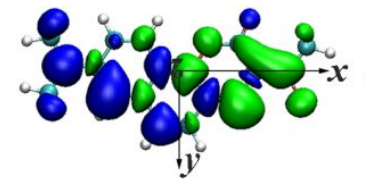

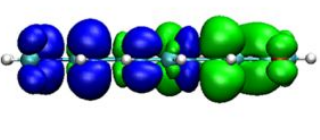

benza2-CO-S1 front view

benza2-CO-S1 side view

Figure S3. Electron density difference analysis during electron excitation (blue and green regions represent decrease and increase in electron density, respectively) 
AOCL 80. hole

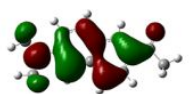

hole

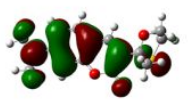

hole

Co

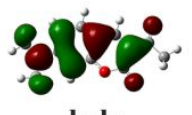

hole

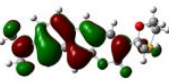

hole

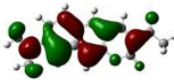

hole

benza1-CO

benza2-COCL $890 \%$ hole

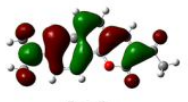

hole
280

electron

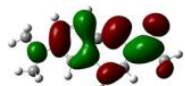

electron

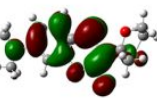

electron

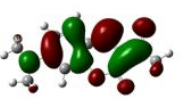

electron

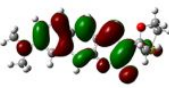

electron

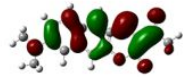

electron

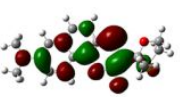

electron

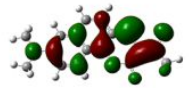

electron

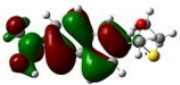

hole

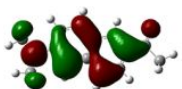

hole

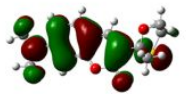

hole

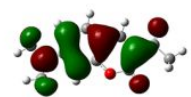

hole

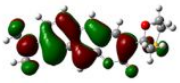

hole

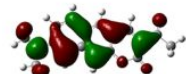

hole

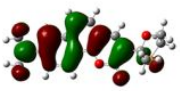

hole

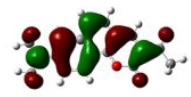

hole

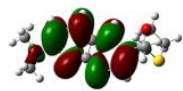

electron

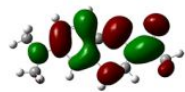

electron

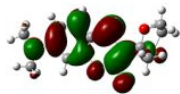

electron

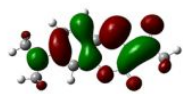

electron

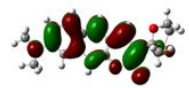

electron

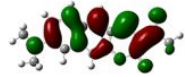

electron

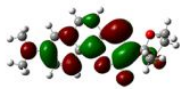

electron

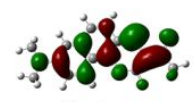

electron

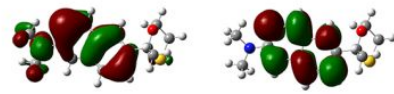

hole

electron
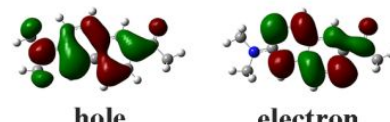

electron
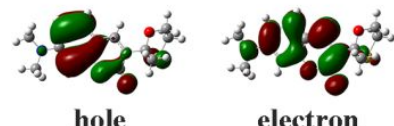

electron
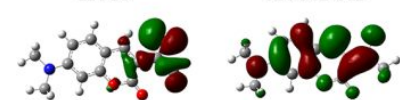

electron
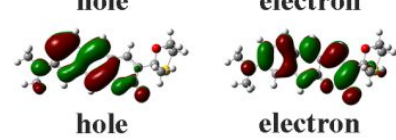

electron
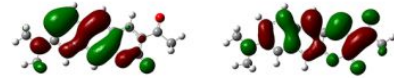

electron

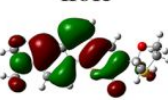

hole

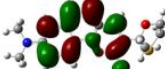

electron
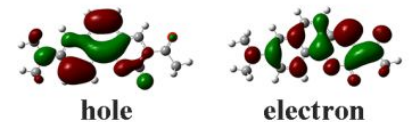

electron

Figure S4. Contour surfaces of the frontier molecular orbitals for investigated compounds in the excited states
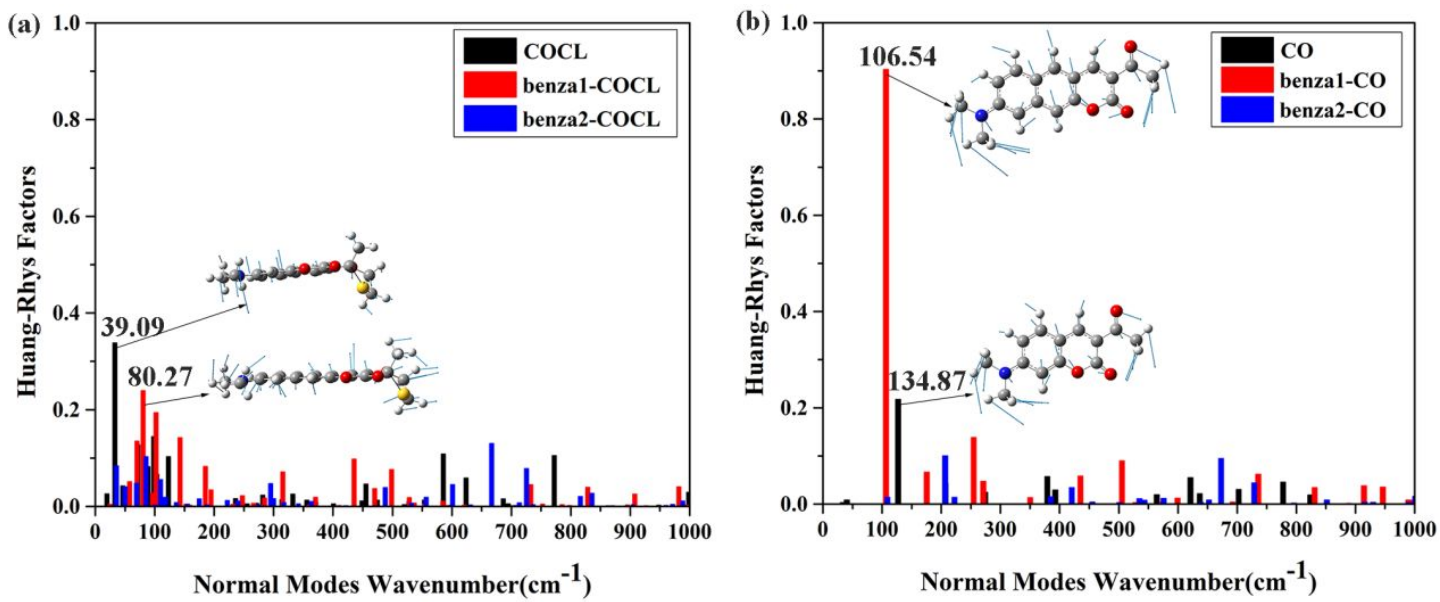

Figure S5. Huang-Rhys (HR) and crucial displacement vectors for the normal modes with large HR in low-frequency regime $\left(<1000 \mathrm{~cm}^{-1}\right)$ for all coumarin-based compounds 
<smiles>CNc1ccc2cc(C3(C)OCCS3)ccc2c1</smiles>

TP-HOCL1<smiles>CC1(c2cc3cc4ccc(N5CCCC5CO)cc4cc3oc2=O)OCCS1</smiles>

rTP-HOCL1<smiles>CNc1ccc2cc(C(C)=O)ccc2c1</smiles>

acedan<smiles>CC(=O)c1cc2cc3ccc(N4CCCC4CO)cc3cc2oc1=O</smiles>

$\mathrm{AcBC} 2$

Figure S6. Structures and corresponding names of the experimental molecules ${ }^{17-18}$

Table S1. Calculated one-photon absorption properties including vertical excitation energy (Evt), maximum absorption peak $\left(\lambda_{\max }\right)$, and corresponding oscillator strength $(f)$ at the ground state geometries of experimental molecules ${ }^{17-18}$ (TP-HOCL1, acedan, rTP-HOCL1 and AcBC2, see Figure S6) by TD-DFT methods using different functionals

\begin{tabular}{|c|c|c|c|c|c|c|}
\hline \multirow[t]{3}{*}{ Molecule } & functional & B3LY & BHandHLYP & CAM-B3LYP & wb97xd & \multirow[t]{3}{*}{ Experimental data } \\
\hline & \multicolumn{5}{|c|}{$\mathrm{P}$} & \\
\hline & $\mathrm{HF} \%$ & 20 & 50 & 19,65 & $22.2,100$ & \\
\hline \multirow[t]{3}{*}{ TP-HOCL1 } & Evt/eV & 3.53 & 4.10 & 3.94 & 3.94 & \multirow{3}{*}{356} \\
\hline & $\lambda_{\max } / n m$ & 351.17 & 302.28 & 314.66 & 314.36 & \\
\hline & $f$ & 0.09 & 0.10 & 0.10 & 0.10 & \\
\hline \multirow[t]{3}{*}{ acedan } & $\mathrm{Evt} / \mathrm{eV}$ & 3.35 & 4.01 & 3.89 & 3.94 & \multirow{3}{*}{356} \\
\hline & $\lambda_{\max } / n m$ & 369.67 & 309.31 & 318.33 & 314.34 & \\
\hline & $f$ & 0.43 & 0.46 & 0.46 & 0.41 & \\
\hline \multirow[t]{3}{*}{ rTP-HOCL1 } & $\mathrm{Evt} / \mathrm{eV}$ & 2.73 & 3.48 & 3.37 & 3.45 & \multirow{3}{*}{424} \\
\hline & $\lambda_{\max } / n m$ & 454.74 & 355.91 & 367.64 & 358.97 & \\
\hline & $f$ & 0.54 & 0.81 & 0.75 & 0.75 & \\
\hline \multirow[t]{3}{*}{$\mathrm{AcBC} 2$} & Evt/eV & 2.47 & 3.13 & 3.08 & 3.15 & \multirow{3}{*}{487} \\
\hline & $\lambda_{\max } / n m$ & 501.89 & 396.15 & 402.53 & 393.20 & \\
\hline & $f$ & 0.67 & 0.94 & 0.91 & 0.91 & \\
\hline
\end{tabular}


Table S2. Calculated two-photon absorption properties including the maximum two-photon absorption cross section $\left(\sigma_{\max }\right)$ and corresponding TPA wavelength $\left(\lambda_{\max }\right)$ at the ground-state geometries of experimental molecules ${ }^{17-18}$ (acedan, rTP-HOCL1 and AcBC2, see Figure S6) by TD-DFT methods using different functionals

\begin{tabular}{llllll}
\hline Molecule & functional & B3LYP & BHandHLYP & $\begin{array}{l}\text { CAM-B3LY } \\
\text { P }\end{array}$ & Experimental data \\
& & & & 19,65 & \\
\cline { 2 - 5 } & HF\% & 20 & 50 & 639.10 & 750 \\
acedan & $\lambda_{\max } / n m$ & 742.43 & 621.48 & 70.60 & 82 \\
& $\sigma_{\max } / \mathrm{GM}$ & 85.00 & 65.50 & & \\
rTP-HOCL & $\lambda_{\max } / n m$ & 911.65 & 742.43 & 735.82 & 900 \\
1 & & & & & \\
& $\sigma_{\max } / \mathrm{GM}$ & 178.00 & 165.00 & 156.00 & 142 \\
& & & & & \\
AcBC2 & $\lambda_{\max } / n m$ & 1012.12 & 794.78 & 818.38 & 900 \\
& $\sigma_{\max } / \mathrm{GM}$ & 212.00 & 265.00 & 279.00 & 439 \\
\hline
\end{tabular}

Table S3. Calculated fluorescent emission properties including vertical excitation energy (Evt), maximum emission peak $\left(\lambda_{\mathrm{ems}}\right)$, and corresponding oscillator strength $(f)$ at the exited state geometries of experimental molecules ${ }^{17-18}$ (acedan, rTP-HOCL1 and AcBC2, see Figure S6) by TD-DFT methods using different functionals

\begin{tabular}{lcccccc}
\hline Molecule & functional & B3LYP & BHandHLYP & CAM-B3LYP & wB97XD & Experimental data \\
\cline { 2 - 5 } & $\mathrm{HF} \%$ & 20 & 50 & 19,65 & $22.2,100$ & \\
\hline acedan & $\mathrm{Evt} / \mathrm{eV}$ & 2.94 & 3.35 & 3.26 & 3.29 & \\
& $\lambda_{\max } / n m$ & 421.31 & 370.34 & 379.98 & 376.86 & 500 \\
& $f$ & 0.62 & 0.76 & 0.78 & 0.74 & \\
rTP-HOCL & $\mathrm{Evt} / \mathrm{eV}$ & 2.25 & 2.78 & 2.68 & 2.76 & \\
1 & & & & & & \\
& $\lambda_{\max } / n m$ & 550.68 & 445.61 & 462.51 & 449.94 & 598 \\
& $f$ & 0.67 & 1.20 & 1.13 & 1.17 & \\
AcBC2 & $\mathrm{Evt} / \mathrm{eV}$ & 2.07 & 2.56 & 2.53 & 2.55 & \\
& $\lambda_{\max } / n m$ & 598.77 & 483.60 & 489.47 & 485.32 & 633 \\
& $f$ & 0.79 & 1.31 & 1.31 & 1.31 & \\
\hline
\end{tabular}

Table S4. Fluorescence properties including fluorescent emission peak $\left(\lambda_{\text {ems }}\right)$, vertical excitation energy $\left(\mathrm{E}_{\mathrm{vt}}\right)$, oscillator strength $(f)$, fluorescent lifetime $(\tau)$ and transition nature of all the studied compounds are calculated by B3LYP/6-311+G (d) in water solvent

\begin{tabular}{lccccccc}
\hline Molecules & $\lambda_{\text {ems }} / n m$ & $\mathrm{Evt} / \mathrm{eV}$ & $f$ & $\tau / n s$ & \multicolumn{3}{c}{ Transition nature } \\
\hline AOCL & 410.67 & 3.11 & 0.14 & 17.98 & $\mathrm{~S}_{1} \rightarrow \mathrm{S}_{0}$ & $\mathrm{H} \rightarrow \mathrm{L}$ & $(97.69 \%)$ \\
AC & 440.97 & 2.95 & 0.64 & 4.56 & $\mathrm{~S}_{1} \rightarrow \mathrm{S}_{0}$ & $\mathrm{H} \rightarrow \mathrm{L}$ & $(98.69 \%)$ \\
COCL & 424.46 & 3.04 & 0.84 & 3.22 & $\mathrm{~S}_{1} \rightarrow \mathrm{S}_{0}$ & $\mathrm{H} \rightarrow \mathrm{L}$ & $(98.77 \%)$ \\
CO & 443.91 & 2.91 & 0.99 & 2.98 & $\mathrm{~S}_{1} \rightarrow \mathrm{S}_{0}$ & $\mathrm{H} \rightarrow \mathrm{L}$ & $(98.99 \%)$
\end{tabular}




\begin{tabular}{llllllll} 
benza1-COCL & 520.51 & 2.46 & 0.71 & 5.73 & $\mathrm{~S}_{1} \rightarrow \mathrm{S}_{0}$ & $\mathrm{H} \rightarrow \mathrm{L}$ & $(98.83 \%)$ \\
benza1-CO & 572.68 & 2.25 & 0.77 & 6.36 & $\mathrm{~S}_{1} \rightarrow \mathrm{S}_{0}$ & $\mathrm{H} \rightarrow \mathrm{L}$ & $(98.15 \%)$ \\
benza2-COCL & 474.27 & 2.72 & 0.87 & 3.87 & $\mathrm{~S}_{1} \rightarrow \mathrm{S}_{0}$ & $\mathrm{H} \rightarrow \mathrm{L}$ & $(99.05 \%)$ \\
benza2-CO & 521.86 & 2.50 & 0.97 & 4.21 & $\mathrm{~S}_{1} \rightarrow \mathrm{S}_{0}$ & $\mathrm{H} \rightarrow \mathrm{L}$ & $(98.74 \%)$ \\
\hline
\end{tabular}

Table S5. The singlet-triplet excited states properties including the adiabatic energy difference between $\mathrm{S}_{1}$ and $\mathrm{T}_{2}\left(\Delta \mathrm{E}_{\mathrm{S} 1-\mathrm{T} 2}\right)$, reorganization energy $\left(\lambda_{\mathrm{S} 1}, \lambda_{\mathrm{T} 2}\right)$ and intersystem crossing rate $\left(K_{i s c}^{S 1-T 2}\right)$ and reverse intersystem crossing rate $\left(K_{i s c}^{S 1-T 2}\right)$ are caculated by B3LYP/6-31G (d) method in water solvent, and spin-orbit coupling matrix elements $\left(H_{\text {soc }}^{S 1-T 2}\right)$ between $\mathrm{S}_{1}$ and $\mathrm{T}_{2}$ states are caculated by using M062X/6-31G (d) method

\begin{tabular}{lccccccc}
\hline Molecules & $\Delta \mathrm{E}_{\mathrm{S} 1-\mathrm{T} 1} / \mathrm{eV}$ & $\Delta \mathrm{E}_{\mathrm{S} 1-\mathrm{T} 2} / \mathrm{eV}$ & $\lambda_{\mathrm{S} 1} / \mathrm{eV}$ & $\lambda_{\mathrm{T} 2} / \mathrm{eV}$ & $H_{\text {Soc }}^{S 1-T 2} / \mathrm{cm}^{-1}$ & $\begin{array}{c}K_{\text {isc }}^{S 1-T 2} \\
/ \mathrm{s}^{-1}\end{array}$ & \begin{tabular}{c}
$K_{\text {risc }}^{T 2} / \mathrm{s}^{-1}$ \\
\hline AOCL
\end{tabular} \\
& 1.11 & 0.29 & 0.28 & 0.40 & 7.52 & $1.66 \times 10^{10}$ & $3.86 \times 10^{5}$ \\
$\mathrm{AC}$ & 0.95 & 0.12 & 0.15 & 0.15 & 0.11 & $6.75 \times 10^{6}$ & $7.40 \times 10^{4}$ \\
$\mathrm{COCL}$ & 0.95 & -0.14 & 0.45 & 0.36 & 9.60 & $4.49 \times 10^{7}$ & $4.59 \times 10^{9}$ \\
CO & 0.89 & 0.11 & 0.32 & 0.31 & 0.07 & $5.77 \times 10^{5}$ & $7.93 \times 10^{3}$ \\
benza1-COCL & 0.76 & -0.03 & 0.15 & 0.16 & 2.88 & $6.56 \times 10^{8}$ & $1.93 \times 10^{9}$ \\
benza1-CO & 0.65 & 0.01 & 0.16 & 0.17 & 0.06 & $4.68 \times 10^{5}$ & $3.43 \times 10^{5}$ \\
benza2-COCL & 0.76 & 0.22 & 0.21 & 0.35 & 5.03 & $7.30 \times 10^{9}$ & $2.29 \times 10^{6}$ \\
benza2-CO & 0.66 & -0.09 & 0.14 & 0.14 & 0.05 & $4.67 \times 10^{4}$ & $1.34 \times 10^{6}$ \\
\hline
\end{tabular}

Table S6. The first triplet excited state $T_{1}$ properties including phosphorescent emission peak $\left(\lambda_{\text {phos }}\right)$, vertical excitation energy $\left(\mathrm{E}_{\mathrm{vt}}\right)$, phosphorescent lifetime $(\tau)$ and transition nature of all the studied compounds are calculated by B3LYP functional in water and 298K, and spin-orbit coupling matrix elements $\mathrm{H}_{\mathrm{soc}}$ between $\mathrm{T}_{1}$ and $\mathrm{S}_{0}$ are calculated at M062X/6-31G(d) theoretical level

\begin{tabular}{lccccccc}
\hline Molecules & $\lambda_{\text {phos }} / n m$ & $\mathrm{Evt} / \mathrm{eV}$ & $\mathrm{H}_{\text {soc }} / \mathrm{cm}^{-1}$ & $\tau_{\text {phos }} / m s$ & \multicolumn{3}{c}{ Transition nature } \\
\hline AOCL & 712.39 & 1.74 & 0.23 & - & $\mathrm{S}_{1} \rightarrow \mathrm{S}_{0}$ & $\mathrm{H} \rightarrow \mathrm{L}$ & $(97.76 \%)$ \\
AC & 661.67 & 1.87 & 0.04 & - & $\mathrm{S}_{1} \rightarrow \mathrm{S}_{0}$ & $\mathrm{H} \rightarrow \mathrm{L}$ & $(91.29 \%)$ \\
COCL & 631.29 & 1.96 & 9.15 & 0.95 & $\mathrm{~S}_{1} \rightarrow \mathrm{S}_{0}$ & $\mathrm{H} \rightarrow \mathrm{L}$ & $(96.24 \%)$ \\
CO & 624.10 & 1.99 & 0.59 & 2.55 & $\mathrm{~S}_{1} \rightarrow \mathrm{S}_{0}$ & $\mathrm{H} \rightarrow \mathrm{L}$ & $(99.02 \%)$ \\
benza1-COCL & 754.68 & 1.64 & 5.23 & 3.35 & $\mathrm{~S}_{1} \rightarrow \mathrm{S}_{0}$ & $\mathrm{H} \rightarrow \mathrm{L}$ & $(92.63 \%)$ \\
benza1-CO & 788.72 & 1.57 & 0.16 & 4.85 & $\mathrm{~S}_{1} \rightarrow \mathrm{S}_{0}$ & $\mathrm{H} \rightarrow \mathrm{L}$ & $(96.98 \%)$ \\
benza2-COCL & 674.57 & 1.84 & 6.72 & 1.09 & $\mathrm{~S}_{1} \rightarrow \mathrm{S}_{0}$ & $\mathrm{H} \rightarrow \mathrm{L}$ & $(91.74 \%)$ \\
benza2-CO & 713.73 & 1.74 & 0.24 & 3.18 & $\mathrm{~S}_{1} \rightarrow \mathrm{S}_{0}$ & $\mathrm{H} \rightarrow \mathrm{L}$ & $(96.74 \%)$ \\
\hline
\end{tabular}

Table S7. TPA tensor elements $\left(S_{a b}\right)$ and TPA cross section $\left(\sigma^{\mathrm{T}}{ }_{\max }\right)$ of all investigated compounds are calculated at B3LYP/6-311+G (d) using DALTON program in water solvent

\begin{tabular}{llccccccc}
\hline Systems & Ex.States & \multicolumn{6}{c}{ TPA tensor elements (in a.u.) } & \multirow{2}{*}{$\sigma^{\mathrm{T}}{ }_{\max } / \mathrm{GM}$} \\
\cline { 2 - 8 } & & $S_{x x}$ & $S_{y y}$ & $S_{z z}$ & $S_{x y}$ & $S_{x z}$ & $S_{y z}$ & \\
\hline AOCL & 1 & 48.80 & -6.70 & -1.00 & -12.80 & -3.70 & 2.50 & 4.32 \\
& 2 & -143.60 & 5.40 & 0.60 & 5.30 & 4.20 & 0.30 & 49.50 \\
AC & 1 & -264.60 & 12.60 & 1.20 & -20.70 & 0.10 & 0.00 & 99.40 \\
COCL & 1 & 158.80 & -0.10 & -0.80 & -11.20 & 14.10 & -1.90 & 40.50 \\
& 2 & -168.50 & 0.40 & -3.90 & 15.10 & 18.30 & -2.30 & 61.90
\end{tabular}




\begin{tabular}{llccccccc} 
CO & 1 & 195.10 & -0.50 & -1.10 & -29.00 & 0.00 & 0.00 & 55.00 \\
benza1-COCL & 1 & 357.90 & -5.30 & -0.40 & -14.70 & -15.80 & 1.50 & 143.00 \\
benza1-CO & 1 & 423.80 & -2.50 & -0.80 & 50.90 & 0.00 & 0.00 & 168.00 \\
benza2-COCL & 1 & -335.80 & 6.50 & 0.30 & 10.60 & -17.10 & 1.20 & 144.00 \\
benza2-CO & 1 & -416.10 & 10.20 & 0.90 & 14.90 & 0.00 & 0.00 & 176.00 \\
\hline
\end{tabular}

Table S8. TPA tensor elements $\left(S_{a b}\right)$ and TPA cross section $\left(\sigma^{\mathrm{T}}{ }_{\max }\right)$ of all investigated compounds are calculated at B3LYP/6-311+G (d) using few-state models in water solvent

\begin{tabular}{lllllllll}
\hline \multirow{2}{*}{ Systems } & \multirow{2}{*}{ Ex.States } & \multicolumn{6}{c}{ TPA tensor elements (in a.u.) } & \multirow{2}{*}{$\sigma_{\text {max }}^{\mathrm{T}}$ /GM } \\
\cline { 3 - 8 } & & $S_{x x}$ & $S_{y y}$ & $S_{z z}$ & $S_{x y}$ & $S_{x z}$ & $S_{y z}$ & \\
\hline AOCL & 1 & -53.17 & 5.73 & -0.55 & 22.33 & 5.41 & -2.45 & 3.00 \\
& 2 & -145.78 & 1.13 & -0.10 & 12.83 & 2.72 & -0.43 & 52.41 \\
AC & 1 & -334.97 & 8.94 & 0.00 & -12.82 & -0.31 & -0.10 & 80.33 \\
COCL & 1 & -234.61 & -2.60 & 0.64 & 29.21 & -9.30 & 2.18 & 44.89 \\
& 2 & -206.58 & -0.70 & 3.53 & 21.49 & 11.42 & -1.44 & 90.21 \\
CO & 1 & -279.60 & -0.37 & 0.00 & 27.24 & 0.00 & 0.00 & 55.78 \\
benza1-COCL & 1 & -510.51 & 4.00 & -0.32 & 43.27 & 16.96 & -2.79 & 146.37 \\
benza1-CO & 1 & 618.72 & -5.27 & 0.00 & 49.93 & 0.00 & 0.00 & 177.23 \\
benza2-COCL & 1 & 485.02 & -0.21 & 0.82 & -17.51 & 20.80 & -0.50 & 151.76 \\
benza2-CO & 1 & 604.85 & -0.02 & 0.00 & 19.79 & 0.00 & 0.00 & 189.90 \\
\hline
\end{tabular}

\section{REFERENCE}

1. Peticolas, W. L. Multiphoton spectroscopy. Annu. Rev. Phys. Chem. 1967, 18, 233-260.

2. Beerepoot, M. T.; Friese, D. H.; List, N. H.; Kongsted, J.; Ruud, K., Benchmarking two-photon absorption cross sections: performance of CC2 and CAM-B3LYP. Phys. Chem. Chem. Phys. 2015, 17, 19306-19314.

3. Rudberg, E.; Salek, P.; Helgaker, T.; Agren, H. Calculations of two-photon charge-transfer excitations using Coulomb-attenuated density-functional theory. $J$. Chem. Phys. 2005, 123, 184108.

4. Monson, P. R.; McClain, W. M. Polarization dependence of the two-photon absorption of tumbling molecules with application to liquid 1-chloronaphthalene and benzene. J. Chem. Phys. 1970, 53, 29-37.

5. Friese, D. H.; Beerepoot, M. T.; Ruud, K. Rotational averaging of multiphoton absorption cross sections. J. Chem. Phys. 2014, 141, 204103.

6. Aidas, K.; Angeli, C.; Bak, K. L.; Bakken, V.; Bast, R.; Boman, L.; Christiansen, O.; Cimiraglia, R.; Coriani, S.; Dahle, P. et al. The Dalton quantum chemistry program system. Wiley Interdiscip. Rev. Comput. Mol. Sci. 2014, 4, 269-284.

7. Lukes, V.; Aquino, A.; Lischka, H. Theoretical study of vibrational and optical spectra of methylene-bridged oligofluorenes. J. Phys. Chem. A 2005, 109, 10232-10238.

8. Peng, Q.; Yi, Y.; Shuai, Z.; Shao, J. Toward quantitative prediction of molecular fluorescence quantum efficiency: role of duschinsky rotation. J. Am. Chem. Soc. 
2007, 129, 9333-9339.

9. Tong, G. S.; Chow, P. K.; To, W. P.; Kwok, W. M.; Che, C. M. A Theoretical investigation into the luminescent properties of $\mathrm{d} 8$-transition-metal complexes with tetradentate schiff base ligands. Chem. Eur. J. 2014, 20, 6433-6443.

10. Englman, R.; Jortner, J. The energy gap law for radiationless transitions in large molecules. Mol. Phys. 1970, 18, 145-164.

11. Lin, S. H. Rate of interconversion of electronic and vibrational energy. J. Chem. Phys. 1966, 44, 3759-3767.

12. Niu, Y.; Peng, Q.; Shuai, Z. Promoting-mode free formalism for excited state radiationless decay process with Duschinsky rotation effect. Sci. China, Ser. B, Chem. 2008, 51, 1153-1158.

13. Niu, Y.; Peng, Q.; Deng, C.; Gao, X.; Shuai, Z. Theory of excited state decays and optical spectra: application to polyatomic molecules. J. Phys. Chem. A 2010, 114, 7817-7831.

14. Shuai, Z.; Peng, Q.; Niu, Y.; Geng, H. MOMAP, Revision 0.3.001; Beijing, China, 2016 (MOMAP: a free and open-source molecular materials property prediction package; avaliable online http://www.shuaigroup.net).

15. Marcus, R. A.; Sutin, N. Electron transfers in chemistry and biology. Biochim. Biophys. Acta, Rev. Bioenerg. 1985, 811, 265-322.

16. Beljonne, D.; Shuai, Z.; Pourtois, G.; Bredas, J. L. Spin-orbit coupling and intersystem crossing in conjugated polymers: A configuration interaction description. J. Phys. Chem. A 2001, 105, 3899-3907.

17. Yuan, L.; Wang, L.; Agrawalla, B. K.; Park, S. J.; Zhu, H.; Sivaraman, B.; Peng, J.; Xu, Q. H.; Chang, Y. T., Development of targetable two-photon fluorescent probes to image hypochlorous acid in mitochondria and lysosome in live cell and inflamed Mouse Model. J. Am. Chem. Soc. 2015, 137, 5930-5938.

18. Jun, Y. W.; Sarkar, S.; Singha, S.; Reo, Y. J.; Kim, H. R.; Kim, J. J.; Chang, Y. T.; Ahn, K. H., A Two-Photon Fluorescent Probe for Ratiometric Imaging of Endogenous Hypochlorous Acid in Live Cells and Tissues. Chem. Commun. 2017, 53, 10800-10803. 